\title{
TORSION FREE AND PROJECTIVE MODULES
}

\author{
BY \\ HYMAN BASS( ${ }^{1}$ )
}

Introduction. Serre has asked [5, p. 243] whether projective modules over the polynomial ring $K\left[X_{1}, \cdots, X_{n}\right], K$ a field, are free, and Seshadri has proved that this is so when $n=2$. More precisely, he showed that every $R[X]$-projective module "comes from" one over $R$ if $R$ is either a principal ideal domain [6] or the coordinate ring of a nonsingular affine curve over an algebraically closed field [7]. By modifying Seshardri's argument in [6] we show here (Theorem 2.4 ) that $R$ may be any Dedekind ring $\left({ }^{2}\right)$. Moreover we provide an example, due to $\mathrm{S}$. Schanuel, which shows that some assumption like integral closure on $R$, is, in general, necessary for this conclusion.

Problems of the above type can often be reduced to showing that a projective module is a direct sum of modules of rank 1 , and this led us to ask for what integral domains $R$ this is true for all (finitely generated) torsion free modules. Our main result (Theorem 1.7) gives an essentially complete solution to this problem. With mild assumptions on $R$, a necessary and sufficient condition is that every ideal in $R$ can be generated by two elements. Thus these integral domains are very close to, but need not be, Dedekind rings.

We assume a familiarity with the basic techniques of commutative algebra as developed in Zariski and Samuel [9]. Homological algebra is appealed to in two discrete instances; one is a reference in the proof of Proposition 1.5, and the other is the proof of Lemma 1.9. The latter result, elementary as it is, seems to defy any proof which does not either use, or essentially reconstruct, the functor Ext ${ }^{1}$.

Minor portions of this work were present in the author's doctoral dissertation. He gratefully records his indebtedness to Professor Kaplansky, who communicated a computation which suggested one of the essential ideas in the proof of Theorem 1.7.

1. Torsion free modules. Throughout this paper "module" means "finitely generated module," and "ring" means "commutative ring." If $M$ is an $R$ module $\mu_{R}(M)$ denotes the least number of elements required to generate $M$, and $\mu^{*}(R)=\sup \mu_{R}(\mathfrak{A})$ where $\mathfrak{A}$ ranges over all finitely generated ideals of $R$. We call $R$ local if it has a unique maximal ideal.

Presented to the Society, January 29, 1960; received by the editors April 8, 1961.

(1) This work has been partially supported by the Office of Naval Research under contract NONR 266(57).

(2) After writing this paper the author discovered that Serre, with slightly different techniques, proved the same extension of Seshadri's theorem, in the Seminaire Dubreil. 
Proposition 1.1. $R$ is local if and only if $\mu_{R}$ is additive; i.e. if and only if $\mu_{R}(M \oplus N)=\mu_{R}(M)+\mu_{R}(N)$ for all $R$-modules $M$ and $N$.

Proof. If $\mathfrak{M}$ and $\mathfrak{R}$ are distinct maximal ideals then $1=\mu_{R}(R / \mathfrak{M} \cap \mathfrak{N})$ $=\mu_{R}(R / \mathfrak{M} \oplus R / \mathfrak{R})$, so $\mu_{R}$ is not additive. Conversely, if $R$ is local, with maximal ideal $\mathfrak{M}$, then by Nakayama's lemma, $\mu_{R}(M)=\mu_{R}(M / \mathfrak{M} M)$ $=\mu_{R / \mathfrak{R}}(M / \mathfrak{M} M)$ for all $R$-modules $M$. Therefore the additivity of $\mu_{R}$ reduces

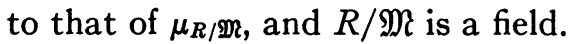

REMARK. Neither the proposition nor the proof above requires that $R$ be commutative.

Now let $R$ be an integral domain with field of quotients $L$, and let $M$ be an $R$-module. Then $\operatorname{rank}_{R} M=\operatorname{dim}_{L} L \otimes_{R} M$. It is easy to see that $\operatorname{rank}_{R} M$ $\leqq \mu_{R}(M)$, with equality if and only if $M$ is free. If $N$ is a submodule of $M$ we call $N$ closed in $M$ if $M / N$ is torsion free. If $S$ is any subset of $M$, the closure of $S$ in $M$ is the smallest closed submodule of $M$ containing $S$.

Proposition 1.2. Let $R$ be an integral domain for which every torsion free $R$-module is a direct sum of modules of ranks at most $k(k \geqq 1)$. Then, for each maximal ideal $\mathfrak{M}$ of $R, \mu^{*}\left(R_{\mathfrak{M}}\right) \leqq k+1$.

Proof. To show that the hypothesis on $R$ is inherited on $R_{\mathfrak{R}}$ we observe that if $A$ is a finitely generated $R_{\mathfrak{R}}$-module, and $B$ is the $R$-submodule of $A$ generated by a finite $R_{\mathfrak{M}}$-generating set of $A$, then $A=R_{\mathfrak{R}} \otimes_{R} B$, so if $B$ splits into a direct sum, so does $A$. Hence, we may assume $R$ is already local.

Suppose there is an ideal $\mathfrak{A}=\sum_{i=1}^{k+2} R a_{i} \subset R$ with $\mu(\mathfrak{I})\left(=\mu_{R}(\mathfrak{A})\right)=k+2$. Let $F=R^{k+2}$ and $\alpha=\left(a_{1}, \cdots, a_{k+2}\right) \in F$. We claim that $\alpha$ belongs to no proper direct summand of $F$. For suppose the contrary. Since $R$ is local projective modules are free, so this amounts to saying that, relative to some new basis for $F, \alpha$ has at least one zero coordinate. But $\mathfrak{A}$ is the ideal generated by the coordinates of $\alpha$ relative to any basis of $F$. E.g. an invariant description of $\mathfrak{A}$ is: $\mathfrak{A}=\left\{f(\alpha) \mid f \in F^{*}\right\}$, where $F^{*}=\operatorname{Hom}_{R}(F, R)$. Therefore, since $\mu(\mathfrak{R})=k+2$, no coordinate of $\alpha$ can vanish.

Now let $K$ denote the closure of $\{\alpha\}$ in $F$, and set $A=F / K$. Then $A$ is torsion free, $\mu(A) \leqq k+2$, and, since rank $K=1(K=\{x \in F \mid r x \in R \alpha$ for some $r \neq 0$ in $R\})$, $\operatorname{rank} A=\operatorname{rank} F-\operatorname{rank} K=k+1$. We claim that $A$ is indecomposable; once shown, this will conclude the proposition.

Suppose $A=B \oplus C$. Then we have, using (1.1),

$$
\begin{aligned}
\mu(B)+\mu(C) & =\mu(A) \leqq k+2, \\
\operatorname{rank} B+\operatorname{rank} C & =\operatorname{rank} A=k+1,
\end{aligned}
$$

and

$$
\text { rank } B \leqq \mu(B), \quad \text { rank } C \leqq \mu(C) .
$$

From these conditions we conclude that either rank $A=\mu(A)$, rank $B=\mu(C)$, or rank $C=\mu(C)$; i.e. either $A, B$, or $C$ is free. $A$ is not free for then $K$ would 
be a proper direct summand of $F$ containing $\alpha$. Suppose, therefore, that $B$, say, is free. Projecting $A$ onto $B$ with kernel $C$ induces a map $f$ of $F$ onto $B$ which thus splits. Now as above, since $\alpha \in \operatorname{ker} f, \operatorname{ker} f=F$, so $B=0$.

The next proposition sharpens a result of Cohen [3, Theorem 10]. We first record a version of the Chinese Remainder Theorem which we shall need.

LEMMA 1.3. If $\mathfrak{A}_{1}, \cdots, \mathfrak{A}_{r}$ are pairwise comaximal ideals in $R$ and $\alpha_{1}, \cdots, \alpha_{r}$ are elements of an $R$-module $A$, then there is an $\alpha \in A$ such that $\alpha \equiv \alpha_{i} \bmod \mathfrak{A}_{i} A, i=1, \cdots, r$.

Proof. By a standard argument (see [9, Chapter III, \$13]) we can find $e_{1}, \cdots, e_{r} \in R$ such that $e_{i} \equiv \delta_{i j} \bmod \mathfrak{A}_{j}$. Then $\alpha=e_{1} \alpha_{1}+\cdots+e_{r} \alpha_{r}$ works.

If $R$ is Noetherian, $K-\operatorname{dim} R$ denotes the Krull dimension of $R$, i.e. the maximum length of a chain of prime ideals in $R$.

Proposition 1.4 (COHEN). If $R$ is a Noetherian integral domain for which $\mu^{*}\left(R_{\mathfrak{M}}\right) \leqq k$ for all maximal ideals $\mathfrak{M}$, then $K-\operatorname{dim} R \leqq 1$ and $\mu^{*}(R) \leqq \max (2, k)$.

Proof. $K-\operatorname{dim} R \leqq 1$ by [3, Corollary 1 to Theorem 10]. Moreover, if $k=1$ then $R$ is a Dedekind ring and the conclusion is well known in this case (see $\left[9\right.$, p. 279]). Hence, we may assume $k \geqq 2$, and we must show $\mu^{*}(R) \leqq k$.

Let $\mathfrak{A}$ be a nonzero ideal in $R$, and let $\mathfrak{M}_{1}, \cdots, \mathfrak{M}_{n}$ be the maximal ideals containing $\mathfrak{A}$; there are only finitely many since $K$-dim $R \leqq 1$. By (1.3) we may choose $\alpha_{1} \in \mathfrak{A}$ so that $\alpha_{1}$ is part of a minimal generating set of $\mathfrak{A} R_{\mathfrak{N}_{\mathfrak{i}}}$, $i=1, \cdots, n$. Now let $\mathfrak{N}_{1}, \cdots, \mathfrak{N}_{r}$ be those maximal ideals containing $\alpha_{1}$. Then we may choose $\alpha_{2 j}, \cdots, \alpha_{k j} \in \mathfrak{A}$ so that $\left\{\alpha_{1}, \alpha_{2 j}, \cdots, \alpha_{k j}\right\}$ generate $\mathfrak{U} R_{\mathfrak{M}_{j}}$, for each $j=1, \cdots, r$. We have arranged this when $\mathfrak{N}_{j}=$ some $\mathfrak{M}_{i}$, and otherwise $\mathfrak{A} R_{\mathfrak{D}_{j}}=R_{\mathfrak{P}_{j}}$ is generated, by a single element of $\mathfrak{A}$. Now, by (1.3) again, we may choose $\alpha_{h} \in \mathfrak{A}$ so that $\alpha_{h} \equiv \alpha_{h j} \bmod \mathfrak{N}_{j} \mathfrak{A}, j=1, \cdots, r$, $h=2, \cdots, k$. Then if $\mathfrak{A}_{0}=\left(\alpha_{1}, \alpha_{2}, \cdots, \alpha_{k}\right), \mathfrak{A}_{0} R_{\mathfrak{N}_{j}}=\mathfrak{A}_{\mathfrak{M}_{j}}, j=1, \cdots, r$, and if $\mathfrak{M} \neq$ any $\mathfrak{N}_{j}, \mathfrak{A}_{0} R_{\mathfrak{D}}=\mathfrak{A} R_{\mathfrak{R}}=R_{\mathfrak{R}}$. Hence $\mathfrak{A}_{0}$ and $\mathfrak{A}$, agreeing locally, are equal.

At this point we have concluded the easier half of our main theorem (Theorem 1.7). We now commence the more difficult task of decomposing certain torsion free modules. The next proposition accomplishes a first reduction of the problem.

Proposition 1.5. If $R$ is a Noetherian integral domain with $\mu^{*}(R) \leqq 2$, then a closed projective submodule of a torsion free $R$-module is a direct summand.

Proof. By [1, Theorem 3.3] the conclusion above is equivalent to the condition that $\mathfrak{A}=\left(\mathfrak{A}^{-1}\right)^{-1}$ for all nonzero ideals $\mathfrak{A}$ in $R$. Since we know that $K$-dim $R \leqq 1$ it therefore suffices to prove

Lemma 1.6. Let $R$ be a Noetherian integral domain with $K$-dim $R \leqq 1$, and let $\mathfrak{B} \subset \mathfrak{A}$ be nonzero ideals with $\mathfrak{A}^{-1}=\mathfrak{B}^{-1}$. Then if $\mu_{R}(\mathfrak{H}) \leqq 2, \mathfrak{A}=\mathfrak{B}$.

Proof. Everything localizes properly, so we may reduce to the case where 
$R$ is local with maximal ideal $\mathfrak{M} \neq 0$. If $\mathscr{A} \neq \mathfrak{B}$ we may, after enlarging $\mathfrak{B}$ if necessary, assume $\mathfrak{A} / \mathfrak{R} \cong R / \mathfrak{M}$. Then $\mathfrak{A}=(a, b)$ with $\mathfrak{B}=(\mathfrak{M} \mathfrak{A}, b), a \in \mathfrak{A}$, and $a \notin \mathfrak{B}$. Since $K$-dim $R \leqq 1, \mathfrak{M}^{K} a \subset b R$ for some $K$. If $K$ is the least such integer then $K \geqq 1$, and we may choose $x \in \mathfrak{M}^{K-1}$ so that $x a \notin b R$; then $\mathfrak{M} x a \subset b R$, and $x b^{-1} \in a^{-1} R \supset \mathfrak{A}^{-1}$. However, since $\mathfrak{B}=(\mathfrak{M} \mathfrak{A}, b)=(\mathfrak{M} a, b), x b^{-1} \in \mathfrak{O}^{-1}$, so $\mathfrak{B}^{-1} \neq \mathfrak{P}^{-1}$; contradiction.

An example at the end of this section shows that the hypotheses of (1.6) are fairly delicate.

We now state our principal theorem.

THEOREM 1.7. Let $R$ be a Noetherian integral domain whose integral closure, $\bar{R}$, is a finite $R$-module. Then every torsion free $R$-module is a direct sum of modules of rank one if and only if $\mu^{*}(R) \leqq 2$. Moreover, in this case every torsion free $R$-module of rank one is a projective $S$-module for a unique ring $S$ with $R \subseteq S \subseteq \bar{R}$.

The proof is an induction on the finite length $l(\bar{R} / R)$. We prepare for the proof with three lemmas. The induction step is accomplished with (1.5) and (1.8) when $R$ is local. Then we use (1.3), (1.9), and (1.10) to pass first to the semi-local, and finally to the general case.

LeMma 1.8. Let $R$ be a Notherian local integral domain with maximal ideal $\mathfrak{M}$ and integral closure $\bar{R}$, and assume $\mu^{*}(R)=2$. Then

(i) $R_{1}=\mathfrak{M}^{-1}$ is a proper finite integral overring of $R$.

(ii) Every nonprincipal ideal $\mathfrak{A}$ is an $R_{1}$-module; i.e. $R_{1} \mathfrak{A}=\mathfrak{A}$.

(iii) If $S$ is a proper finite integral overring of $R$ then $R_{1} \subseteq S$ and $\mu^{*}(S) \leqq 2$.

Proof. $\mathfrak{M} \subset \mathfrak{M M}^{-1} \subset R$ so $\mathfrak{M M}^{-1}=\mathfrak{M}$ or $R$. If $\mathfrak{M M}^{-1}=R$, $\mathfrak{M}$ is invertible, so principal, $R$ is a discrete valuation ring, and $\mu^{*}(R)=1$. Therefore, since $\mu^{*}(R)=2, \mathfrak{M M}^{-1}=\mathfrak{M}$, and from this it follows that $R_{1}=\mathfrak{M}^{-1}$ is a ring. Moreover, since $K$-dim $R=1, \mathfrak{M}$ is a prime of a principal ideal, so $R_{1} \neq R$ (see [9, proof of Lemma 6, p. 276]). $R_{1}$ is obviously a finite $R$-module, so we have established (i).

Let $\mathscr{P}$ be a nonprincipal fractional ideal of $R$ and let $\mathscr{B}=\mathfrak{M}^{-1} \mathscr{\mathscr { A }}$. Then $\mathfrak{M P}=\mathfrak{M M}^{-1} \mathfrak{I}=\mathfrak{M} \mathfrak{A}$. Now $\mathfrak{A}=(a, b)$ with $a$ and $b$ independent modulo $\mathfrak{M} \mathfrak{A}$. Therefore $a$ and $b$, being elements of $\mathfrak{B}$ independent modulo $\mathfrak{M} \mathscr{B}$, generate $\mathfrak{B}$, since $\mu_{R}(\mathfrak{B}) \leqq 2$. Thus $\mathfrak{B}=\mathfrak{A}$, and this proves (ii).

Finally, (iii) follows by applying (ii) to $\mathfrak{A}=S$. The fact that $\mu^{*}(S) \leqq 2$ comes from viewing $S$-ideals as $R$-ideals.

Lemma 1.9. If $R$ is a commutative Noetherian ring and $0 \rightarrow A \rightarrow B \rightarrow C \rightarrow 0$ an exact sequence of (finitely generated) $R$-modules, then the sequence splits if and only if it splits locally.

Proof. The finiteness assumptions guarantee that $\operatorname{Ext}_{R}^{1}(C, A)$ localizes properly; i.e. $\operatorname{Ext}_{R}^{1}(C, A)_{\mathfrak{N}}=\operatorname{Ext}_{R \mathfrak{n}}^{1}\left(C_{\mathfrak{N}}, A_{\mathfrak{N}}\right)$. Viewing the exact sequence as an element of $\operatorname{Ext}_{R}^{1}(C, A)$, therefore, the lemma reduces to the familiar 
fact that an element of an $R$-module is zero if and only if it becomes zero at all localizations.

Lemma 1.10. Let $R$ be a local Noetherian integral domain with maximal ideal $\mathfrak{M}$, and let $K-\operatorname{dim} R=1$. Suppose $A$ is a torsion free $R$-module and $\alpha$ is a nonzero element of $A$ for which the closure of $\{\alpha\}$ in $A$ is a direct summand. Then there exists an integer $n>0$ such that if $\alpha^{\prime} \equiv \alpha \bmod \mathfrak{M}^{n} A$, the closure of $\left\{\alpha^{\prime}\right\}$ in $A$ is a direct summand.

Proof. Write $A=A_{0} \oplus B$ with $A_{0}$ the closure of $\{\alpha\}$ in $A ; A_{0}=\{\gamma \in A \mid$ $r \gamma \in R \alpha$ for some $r \neq 0$ in $R\}$. Choose $a \neq 0$ in $R$ so that $a A_{0} \subset R \alpha$; this is clearly possible. Then take $n$ large enough so that $\mathfrak{M}^{n} \subset a \mathfrak{M}$; this is possible because, $K$ - $\operatorname{dim} R$ being one, every nonzero ideal is $\mathfrak{M}$-primary. Now suppose $\alpha^{\prime}=\alpha+\beta$ with $\beta \in \mathfrak{M}^{n} A$. Write $\beta=\beta_{0}+\beta_{1}, \beta_{0} \in A_{0}, \beta_{1} \in B$. Then $\beta_{0} \in \mathfrak{M}^{n} A_{0} \subset \mathfrak{M a} A_{0}$ $\subset \mathfrak{M R} \alpha=\mathfrak{M} \alpha$, so $\beta_{0}=m \alpha$ for some $m \in \mathfrak{M}$, and $\alpha+\beta_{0}=(1+m) \alpha$. Since $1+m$ is a unit there is no harm in replacing $\alpha$ by $(1+m) \alpha$, in which case we may assume $\beta=\beta_{1} \in B$. Let $A_{0}^{\prime}$ be the closure of $\left\{\alpha^{\prime}\right\}$ in $A$. Since $\alpha \neq 0$ it is clear that $A_{0}^{\prime} \cap B=0$, so if $A^{\prime}=A_{0}^{\prime}+B$, then actually $A^{\prime}=A_{0}^{\prime} \oplus B$.

We conclude by showing that $A^{\prime}=A$, for which it suffices to show that $A_{0} \subset A^{\prime}$. Let $\gamma \in A_{0}$ then $a \gamma=r \alpha$ for some $r \in R$. Write $\beta=a \beta^{\prime}$ (recall $\beta \in \mathfrak{M}^{n} A$ $\subset a \mathfrak{M} A(a A)$. Then $a\left(\gamma+r \beta^{\prime}\right)=a \gamma+r a \beta^{\prime}=r \alpha+r \beta=r \alpha^{\prime} ;$ thus $\gamma+r \beta^{\prime} \in A_{0}^{\prime}$ $\subset A^{\prime}$. But since $B$ is closed in $A$, and $a \beta^{\prime}=\beta \in B$, we have $\beta^{\prime} \in B$. Therefore $r \beta^{\prime} \in B \subset A^{\prime}$, so, finally, $\gamma \in A_{0}^{\prime}+B=A^{\prime}$.

We shall now prove Theorem 1.7 , by induction on $l(\bar{R} / R)$, the case $\bar{R}=R$ being the classical theory of Steinitz-Chevalley (see [4]) for Dedekind rings. As has been pointed out above, one of the implications follows immediately from (1.2) and (1.4). Our task now is to show that if $A$ is a nonzero torsion free $R$-module, then $A$ is a direct sum of modules of rank one. We proceed in three steps: local, semi-local, and general.

Local case. We may assume $A$ has no nonzero free direct summands (after removing any that exist). Let $A_{0}$ be a closed submodule of $A$ of rank 1 ; then $A_{0}$ is isomorphic to an ideal in $R$. If $A_{0}$ is cyclic it is free, so, by (1.5), $A_{0}$ is a free direct summand of $A$, contrary to our assumption above. Hence, $A_{0}$ is isomorphic to a nonprincipal ideal of $R$, so, by (ii) of (1.8), $A_{0}$ is actually an $R_{1}$-module, where $R_{1}=\mathfrak{M}^{-1}, \mathfrak{M}$ being the maximal ideal of $R$. Since every element of $A$ belongs to a closed submodule of rank one it follows that $A$ itself enjoys the structure of an $R_{1}$-module. Moreover, by (i) of $(1.8), l_{R_{1}}\left(\bar{R} / R_{1}\right)$ $<l_{R}(\bar{R} / R)$, so we finish by induction.

REMARK. This part of the proof does not establish our result for local rings, since $R_{1}$ need only be semi-local.

Semi-local case. It suffices to show that $A$ has a direct summand of rank one. Let $\mathfrak{M}_{1}, \cdots, \mathfrak{M}_{r}$ be the maximal ideals of $R$. By the local case we may choose an $\alpha_{i} \in A$ such that the closure of $\left\{\alpha_{i}\right\}$ in the $R_{\mathfrak{M}_{i}}$-module $A_{\mathfrak{M}_{i}}$ is a direct summand. By (1.10) there is an $n_{i}>0$ such that $\alpha \equiv \alpha_{i} \bmod \mathfrak{M}_{i}^{n_{i}} A_{\mathfrak{M}_{i}}$ implies the closure of $\{\alpha\}$ in $A_{\mathfrak{N}_{i}}$ is a direct summand of $A_{\mathfrak{M}_{i}}$. Now, by (1.3), 
we may choose $\alpha=A$ so that $\alpha \equiv \alpha_{i} \bmod \mathfrak{M}_{i}^{n_{i}} A, i=1, \cdots, r$. Let $A_{0}$ be the closure of $\{\alpha\}$ in $A$. Since closure commutes with localization the exact sequence $0 \rightarrow A_{0} \rightarrow A \rightarrow A / A_{0} \rightarrow 0$ splits locally; hence, by (1.9), it splits.

General case. Let $\mathfrak{M}_{1}, \cdots, \mathfrak{M}_{r}$ be those primes containing the conductor from $\bar{R}$ to $R$, and let $S=R-\bigcup_{i=1}^{r} \mathfrak{M}_{\imath}$. By the semi-local case we may choose $\alpha \neq 0$ in $A$ so that the closure of $\{\alpha\}$ in $A_{S}$ is a direct summand of the $R_{S^{-}}$ module $A_{s}$. Let $A_{0}$ be the closure of $\{\alpha\}$ in $A$. As above it suffices to show that the exact sequence $0 \rightarrow A_{0} \rightarrow A \rightarrow A / A_{0} \rightarrow 0$ splits locally. We have arranged this at each $\mathfrak{M}_{i}, i=1, \cdots, r$, and at any other $\mathfrak{M}, R_{\mathfrak{R}}$ is a discrete valuation ring, so $\left(A / A_{0}\right) \mathfrak{m}$ is free.

Now we come to the final statement in the theorem. A torsion free $R$ module of rank one is isomorphic to an ideal $\mathfrak{A} \subset R$; we must show that $\mathfrak{A}$ is a projective $S$-module for a unique ring $S, R \subset S \subset \bar{R}$. Again we induce on $l_{R}(\bar{R} / R)$. Write $\mathfrak{A}=\prod_{i} \mathfrak{Q}_{i}$ as a product of primary ideals, and let $\mathfrak{M}_{i}$ denote the radical of $\mathfrak{Q}_{i}$. If $\mathfrak{A}$ is invertible $\mathfrak{A}$ is $R$-projective; otherwise some $\mathfrak{Q}_{i}$ is not invertible. Then, as in the proof of (1.8), $\mathfrak{M}_{i}^{-1}$ is a proper integral overring of $R$, and $\mathfrak{Q}_{i}$ is an $\mathfrak{M}_{i}^{-1}$-module. But then $\mathfrak{M}_{i}^{-1} \mathfrak{A}=\mathfrak{M}_{i}^{-1} \prod_{j} \mathfrak{Q}_{j}=\prod_{j \neq i} \mathfrak{Q}_{j} \mathfrak{M}_{i}^{-1} \mathfrak{Q}_{i}$ $=\mathfrak{A}$, so $\mathfrak{A}$ is an $\mathfrak{M}_{i}^{-1}$-module, and the existence of $S$ follows by induction. Now if $L$ is the field of quotients of $S$ and $\mathfrak{A} \subset I$ is a nonzero projective $S$-module, then one can show easily that $S=\{x \in L \mid x \mathfrak{A} \subset \mathfrak{A}\}$. This shows that $S$ is unique.

We shall now consider some examples to which Theorem 1.7 applies. A very accessible one, suggested by Kaplansky, is the ring of Gaussian integers with even imaginary part. In this case there are only two isomorphism types of ideals, represented by (1), and $(2,2 \mathrm{i})$.

An interesting class of rings is obtained as follows. Let $\Sigma$ be an additive submonoid of the non-negative integers, with greatest common divisor 1 . With $K$ a field, let $K^{\Sigma}$ denote the subring of $K[[T]]$ consisting of all power series for which $T^{n}$ has nonzero coefficient only if $n \in \Sigma$. Then $K^{\Sigma}$ is a onedimensional complete local integral domain with integral closure $K[[T]]$. Any such $\Sigma$ is finitely generated as a monoid, and the minimal number of generators of $\Sigma$ is the same as that of the maximal ideal in $K^{\Sigma}$. The relevant observation here is that $\mu^{*}\left(K^{\Sigma}\right) \leqq k$ if and only if $k \in \Sigma$. Thus, the examples subject to Theorem 1.7 are those for which $\Sigma$ is generated by $\{2,2 n+1\}$, $n \geqq 0$. If $n>0$ the inverse of the maximal ideal in $K^{\Sigma}$ is $K^{\Sigma^{\prime}}$, where $\Sigma^{\prime}$ is generated by $\{2,2(n-1)+1\}$. An example which exhibits the necessity of the hypotheses in (1.6) is $K^{\Sigma}$ with $\Sigma$ generated by $\{6,10,15\}$. For in this ring $\left(T^{6}, T^{10}\right)^{-1}=\left(1, T^{29}\right)=\left(T^{6}, T^{10}, T^{15}\right)^{-1}$.

Finally we remark that one should be able to generalize parts of Theorem 1.7 by dropping the requirement that $R$ be an integral domain. While the specific formulation of such a generalization is not clear it should at least include the structure theorem of Reiner [4] for integral representations of cyclic groups of prime order, and perhaps even yield a similar result for more general cyclic groups. 
2. Projective modules over $R[X]$. Throughout this section $R$ denotes a Noetherian integral domain with field of quotients $K$, and $R[X]$ is a polynomial ring over $R$ in the variable $X$. All modules are finitely generated over their designated ring of operators, and $\otimes=\otimes_{R}$. An $R[X]$-module $M$ will be called extended if $M \cong R[X] \otimes M_{0}$ for an $R$-module $M_{0}$. If $S$ is a ring and $M$ is an $S$-module, $M^{*}=\operatorname{Hom}_{S}(M, S)$, and we call $M$ reflexive if $M \cong M^{* *}$. Clearly projective modules are reflexive.

Proposition 2.1. (a) If $R$ is integrally closed then every reflexive $R[X]$ module of rank 1 is extended.

(b) (Schanuel) If there is an element $t \in K, t \notin R$, such that $t^{n} \in R$ for all large $n$, then there is a projective $R[X]$-module of rank 1 which is not extended.

Proof. (a) Let $P$ be a reflexive $R[X]$-module of rank 1. Then $P \subset K \otimes P$ which is a free $K[X]$-module of rank 1 . We may choose a generator $\alpha$ of $K \otimes P$ so that $P \subset R[X] \alpha$. It is then clear that for some $a \neq 0$ in $R, a \alpha \in P$. Now, identifying $R[X] \alpha$ with $R[X]$ we may assume that $P$ is an ideal in $R[X]$ and that (i) $P_{0}=P \cap R \neq 0$. Since $R$ is integrally closed so is $R[X]$, and since $P$ is reflexive, $P=\left(P^{-1}\right)^{-1}$. It follows then (see $[8, \S 105]$ ) that (ii) $P$ is unmixed of height 1.

From (i) and (ii) above we shall prove that $P=P_{0} R[X] \cong R[X] \otimes P_{0}$. For consider all ideals $\mathfrak{A}$ in $R[X]$ such that $\mathfrak{A}=\mathfrak{A}_{0} R[X]$, where $\mathfrak{A}_{0}=\mathfrak{A} \cap R$. They are closed under intersection. Hence it suffices to treat a primary component $\mathfrak{Q}$ of $P$, say with radical $\mathfrak{B}$. Now $\mathfrak{B}_{0}=\mathfrak{B} \cap R \supset P_{0} \neq 0$ so since $h t \mathfrak{B}$ $=1, \mathfrak{B}=\mathfrak{B}_{0} R[X]$. Finally the only $\mathfrak{B}$-primary ideals are $\mathfrak{P}^{(n)}$ (see $[8$, Chapter IV, 12]), $n>0$, and it is easy to see that $\mathfrak{B}^{(n)}=\mathfrak{B}_{0}^{(n)} R[X]$.

(b) Suppose $t \in K, t \notin R$, and $t^{n} \in R$ for all large $n$. Replacing $t$ by a power of $t$, if necessary, we may assume $t^{n} \in R$ for all $n \geqq 2$. Now set $a=t X$ and consider the fractional ideals

$$
\mathfrak{A}=\left(1+a, 1+a+a^{2}\right)
$$

and

$$
\mathfrak{B}=\left(1-a+a^{2}, 1-a\right) .
$$

Then $\mathfrak{A} \mathfrak{B}=\left(1+a^{3}, 1-a^{2}, 1+a^{2}+a^{4}, 1-a^{3}\right)$. Therefore, since $a^{n} \in R[X]$ for all $n \geqq 2$, and since $1=-a^{2}\left(1+a^{3}\right)+a^{2}\left(1-a^{2}\right)+\left(1+a^{2}+a^{4}\right)-a^{2}\left(1-a^{3}\right)$, we have $\mathfrak{A} \mathfrak{B}=R[X]$ so $\mathfrak{A}$ is invertible with $\mathfrak{B}=\mathfrak{A}^{-1}$.

Now we contend that $\mathfrak{A}$ is not isomorphic to an extended ideal. For suppose it is. Choose a prime $\mathfrak{B}$ in $R$ so that $t \in R_{\mathfrak{B}}$. Then, by passing to $R_{\mathfrak{B}}$ and $R_{\mathfrak{B}}[X]$ we can assume $R$ is local. In this case an extended invertible ideal must be principal, so $\mathfrak{A}=(c)$ for some $c \in \mathfrak{A}$. Since $\mathfrak{A} \subset K[X]$ and $\mathfrak{A} K[X]=K[X]-$ $1=\left(1+a+a^{2}\right)-a(1+a)$-we must have $c \in K$. Now $c^{-1}(1+a)=c^{-1}+c^{-1} t X$ $\in R[X]$ so $c^{-1} \in R$. Therefore, $\left(c^{-1}\right)=\mathfrak{A}^{-1}=\mathfrak{B} \subset R[X]$; but manifestly $\mathfrak{B} ণ R[X]$-contradiction. 
All the examples discussed at the end of the last section furnish instances fulfilling the hypotheses of part (b) above.

We proceed now to the generalization of Seshadri's results. The essential idea is contained in the next proposition, which we have extracted from Seshadri's argument. It is slightly reminiscent of the Steinitz theorem which asserts that, over a Euclidean ring, bases for a free module and free submodule may be "lined up" properly, so to speak. Curiously, the formulation below does not even require $R$ to be Noetherian.

Seshadri proved our Lemma 2.3 with the use of matrices, and this (alone) confined his theorem to principal ideal rings.

Proposition 2.2. Let $\mathfrak{B}_{0}$ be a maximal ideal in $R, P$ an $R[X]$-module for which $P / \mathfrak{B}_{0} P$ is a torsion free (and therefore free) $R / \Re_{0}[X]$-module, and let $L=L_{1} \oplus \cdots \oplus L_{n} \subset P$ with each $L_{i} R[X]$-projective of rank 1 . Then there exists an automorphism $\alpha$ of $L$ and an integer $r, 0 \leqq r \leqq n$, such that $\alpha\left(L_{1} \oplus \cdots \oplus L_{r}\right) \subset \mathfrak{B}_{0} P$ and

$$
\alpha\left(L_{r+1} \oplus \cdots \oplus L_{n}\right) \cap \mathfrak{B}_{0} P=\mathfrak{B}_{0} \cdot \alpha\left(L_{r+1} \oplus \cdots \oplus L_{n}\right) .
$$

The proof will be preceded by a lemma. We denote the residue of anything modulo $\Re_{0}$ by "priming" it. Thus $L$ ' is a free module over the Euclidean ring $R^{\prime}[X]$. Hence, any endomorphism of $L^{\prime}$ has a determinant. In particular the group Aut $\left(L^{\prime}\right)$ of automorphisms of $L^{\prime}$ contains the subgroup, $\operatorname{Aut}_{1}\left(L^{\prime}\right)$, of automorphisms of determinant 1 . These groups are $G L\left(n, R^{\prime}[X]\right)$ and $S L\left(n, R^{\prime}[X]\right)$, respectively.

Lemma 2.3. The natural map $L \rightarrow L^{\prime}$ induces a homomorphism $\phi:$ Aut $(L)$ $\rightarrow \operatorname{Aut}\left(L^{\prime}\right)$, and $\operatorname{Aut}_{1}\left(L^{\prime}\right) \subset \operatorname{im} \phi$.

Proof. $L^{\prime}=L_{1}^{\prime} \oplus \cdots \oplus L_{n}^{\prime}$. Since $R^{\prime}[X]$ is a Euclidean ring $\operatorname{Aut}_{1}\left(L^{\prime}\right)$ is generated by automorphisms of the form $\alpha=1_{L^{\prime}}+f^{\prime}$, where $f^{\prime}\left(L_{k}^{\prime}\right)=0$ for $k \neq i$, and $f^{\prime}\left(L_{i}^{\prime}\right) \subset L_{j}^{\prime}$ for some $i$ and $j, i \neq j$. Since $L_{i}$ is projective we can find a map $g: L_{i} \rightarrow L_{j}$ making

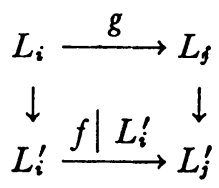

commutative. If $f: L \rightarrow L$ is defined by $f\left(L_{k}\right)=0$ for $k \neq i$, and $f \mid L_{i}=g$, then $f$ reduces modulo $\mathfrak{B}_{0}$ to $f^{\prime}$. Now clearly $\alpha=1_{L}+f$ covers $\alpha^{\prime}$. We need only observe that $\alpha$ is an automorphism. But $\alpha^{-1}=1_{L}-f$ (note that $f^{2}=0$ ).

Proof of (2.2). The inclusion $L \subset P$ induces a map $f: L^{\prime} \rightarrow P^{\prime}$. Since $P^{\prime}$ is free and $R^{\prime}[X]$ is a Euclidean ring im $f \subset P^{\prime}$ is also free, so $f$ splits. Thus $L^{\prime}=\operatorname{ker} f \oplus \operatorname{im} f$. If $\operatorname{rank} \operatorname{ker} f=r$ then we may choose an automorphism $\alpha^{\prime}$ of $L^{\prime}$ of determinant 1 so that $\alpha^{\prime}\left(L_{1}^{\prime} \oplus \cdots \oplus L_{r}^{\prime}\right)=\operatorname{ker} f$. By (1.3) we may lift 
$\alpha^{\prime}$ to an automorphism $\alpha$ of $L$. Then $f^{\prime}$ is zero on $\alpha\left(L_{1} \oplus \cdots \oplus L_{r}\right)^{\prime}$, and a monomorphism on $\alpha\left(L_{r+1} \oplus \cdots \oplus L_{n}\right)^{\prime}$, and from this our conclusion follows.

Theorem 2.4. Let $R$ be a Dedekind ring and $P$ an $R[X]$-module such that $P / \mathfrak{B}_{0} P$ is a torsion free $R / \mathfrak{B}_{0}[X]$-module for all primes $\mathfrak{B}_{0}$ in $R$. Then there is a projective $R$-module $P_{0}$ such that $P \cong R[X] \otimes P_{0}$. In particular, $P$ is projective.

Proof. Taking $\mathfrak{B}_{0}=(0) P$ is torsion free, so $P \subset K \otimes P$, which is a free $K[X]$-module. Choose a basis $\alpha_{1}, \cdots, \alpha_{n}$ for $K \otimes P$ in $P$, and let $L=R[X] \alpha_{1}$ $\oplus \cdots \oplus R[X] \alpha_{n} \subset P$. We have thus established the existence of a submodule $L$ of $P$ for which

(i) $L=L_{1} \oplus \cdots \oplus L_{n}$ with each $L_{i}$ an extended $R[X]$-projective module of rank 1 ; and

(ii) $a P \subset L$ for some $a \neq 0$ in $R$.

Since $P$ is Noetherian we may choose $L$ maximal satisfying (i) and (ii); the theorem then asserts that $L=P$. Suppose not; then $\mathscr{A}_{0}=\{a \in R \mid a P \subset L\}$ $\neq R$. Choose a prime $\mathfrak{B}_{0}$ dividing $\mathfrak{\mathscr { V }}_{0}$. Since $\mathfrak{A}_{0} \neq 0 \mathfrak{\Re}_{0}$ must be maximal $(R$ is a Dedekind ring). Moreover, $\mathfrak{B}_{0} P \cap L \neq \mathfrak{B}_{0} L$. For write $\mathfrak{A}_{0}=\mathfrak{B}_{0} \mathfrak{B}_{0}$; then $\mathfrak{B}_{0} P \nsubseteq L$, but $\mathfrak{B}_{0} \mathfrak{B}_{0} P \subset L$. If $\mathfrak{B}_{0} \mathfrak{B}_{0} P \subset \mathfrak{B}_{0} L$ then $\mathfrak{B}_{0} P \subset \mathfrak{P}_{0}^{-1} \mathfrak{B}_{0} L=L$; contradiction. Therefore $\mathfrak{B}_{0} \mathfrak{B}_{0} P \subset \mathfrak{B}_{0} P \cap L, \mp \mathfrak{B}_{0} L$.

Now we apply (1.2) to obtain an automorphism $\alpha$ of $L$ for which $\alpha\left(L_{1} \oplus \cdots \oplus L_{r}\right) \subset \mathfrak{B}_{0} P$ and

$$
\mathfrak{B}_{0} P \cap \alpha\left(L_{r+1} \oplus \cdots \oplus L_{n}\right)=\mathfrak{B}_{0} \cdot \alpha\left(L_{r+1} \oplus \cdots \oplus L_{n}\right) .
$$

By the remark above, $r>0$. Hence if

$$
H=\mathfrak{B}_{0}^{-1} \cdot \alpha\left(L_{1} \oplus \cdots \oplus L_{r}\right) \oplus \alpha\left(L_{r+1} \oplus \cdots \oplus L_{n}\right),
$$

then $H$ contradicts the maximality of $L$.

\section{REFERENCES}

1. H. Bass, Injective dimension in Noetherian rings, Trans. Amer. Math. Soc. 102 (1962), 18-29.

2. C. Chevalley, L'arithmétique dans les algèbres de matrices, Actualités Sci. Ind., No. 323 Hermann, Paris, 1936.

3. I. S. Cohen, Commutative rings with restricted minimum condition, Duke Math. J. 17 (1950), 27-42.

4. I. Reiner, Integral representations of cyclic groups of prime order, Proc. Amer. Math. Soc. 8 (1957), 142-146.

5. J. P. Serre, Faisceaux algebriques coherents, Ann. of Math. 61 (1955), 197-278.

6. C. S. Seshadri, Triviality of vector bundles over the affine space $K^{2}$, Proc. Nat. Acad. Sci. U.S.A. 44 (1958), 456-458.

7. - Algebraic vector bundles over the product of an affine curve and the affine line, Proc. Amer. Math. Soc. 10 (1959), 670-673.

8. B. L. van der Waerden, Modern algebra, Vol. II, Ungar, New York, 1950.

9. O. Zariski and P. Samuel, Commutative algebra, Vol. I, Van Nostrand, Princeton, N. J., 1958.

Columbia University,

NEw YoRk, NEw YoRk 